\title{
A problem on coextensions
}

\author{
HIDEAKI ŌSHIMA*
}

\begin{abstract}
We raise a problem on coextensions and find partial answers.
\end{abstract}

\section{Introduction}

The Toda bracket is an important tool in homotopy theory. We raise two elementary problems about the Toda bracket and give partial answers for one of them.

Throughout the paper we suppose that a non negative integer $n$ and three pointed maps $Z \stackrel{e}{\longleftarrow} \Sigma^{n} Y=Y \wedge \mathrm{S}^{n}, Y \stackrel{f}{\longleftarrow} X \stackrel{g}{\longleftarrow} W$ are given such that $e \circ \Sigma^{n} f$ and $f \circ g$ are null homotopic $\left(\wedge\right.$ denotes the smash product, that is, $A \wedge B=(A \times B) /\left(A \times\left\{b_{0}\right\} \cup\right.$ $\left.\left.\left\{a_{0}\right\} \times B\right)\right)$.

We shorten the words "with respect to" to "w.r.t.". An extension of $e$ w.r.t. $\Sigma^{n} f$ is a map $\bar{e}: \Sigma^{n} Y \cup_{\Sigma^{n} f} C \Sigma^{n} X \rightarrow Z$ such that $\left.\bar{e}\right|_{\Sigma^{n} Y}=e$. We denote the set of homotopy classes of extensions of e w.r.t. $\Sigma^{n} f$ by $\operatorname{Ext}_{\Sigma^{n} f}(e)$. An $n$-fold coextension of $g$ w.r.t. $f$ is a map $h: \Sigma^{n+1} W=\Sigma \Sigma^{n} W \rightarrow \Sigma^{n} Y \cup_{\Sigma^{n} f} C \Sigma^{n} X$ such that $h\left(w \wedge s_{n} \wedge \bar{t}\right)=$ $\left\{\begin{array}{ll}g(w) \wedge s_{n} \wedge(1-2 t) & 0 \leq t \leq 1 / 2 \\ A(w \wedge(2 t-1)) \wedge s_{n} & 1 / 2 \leq t \leq 1\end{array}\left(w \in W, s_{n} \in \mathrm{S}^{n}, t \in I\right)\right.$ for some null homotopy $A: C W=W \wedge I \rightarrow Y$ of $f \circ g$, where $\bar{t}=(\cos 2 \pi t, \sin 2 \pi t) \in \mathrm{S}^{1}$ and the base points of the unit interval $I=[0,1]$ and the unit circle $S^{1}$ are 1 and $(1,0)$, respectively. A 0 -fold coextension is the same as a coextension defined in [T2, p.13]. We denote the set of homotopy classes of $n$-fold coextensions of $g$ w.r.t. $f$ by $\operatorname{Coext}_{f}^{n}(g)$. When $n=0$, we abbreviate $\operatorname{Coext}_{f}^{n}(g)$ to $\operatorname{Coext}_{f}(g)$.

The set $\{e, f, g\}_{n}=\operatorname{Ext}_{\Sigma^{n} f}(e) \circ \operatorname{Coext}_{f}^{n}(g)$ is called the Toda bracket which is a subset of $\left[\Sigma^{n+1} W, Z\right]$.

If a map $h: \Sigma^{n+1} W \rightarrow \Sigma^{n} Y \cup_{\Sigma^{n} f} C \Sigma^{n} X$ is an $n$-fold coextension of $g$ w.r.t. $f$, then $q_{\Sigma^{n} f} \circ h$ is homotopic to $-\Sigma^{n+1} g$, where $q_{\Sigma^{n} f}: \Sigma^{n} Y \cup_{\Sigma^{n} f} C \Sigma^{n} X \rightarrow \Sigma^{n+1} X$ is the map pinching $\Sigma^{n} Y$ to a point. We denote by $\operatorname{Coext}_{f}^{n}(g)^{*}$ the set of homotopy classes of maps $h: \Sigma^{n+1} W \rightarrow \Sigma^{n} Y \cup_{\Sigma^{n} f} C \Sigma^{n} X$ such that $q_{\Sigma^{n} f} \circ h$ is homotopic to $-\Sigma^{n+1} g$. Obviously $\operatorname{Coext}_{f}^{n}(g) \subset \operatorname{Coext}_{f}^{n}(g)^{*}$. First problem is

Received 30 October 2018; revised 8 February 2019 2010 Mathematics Subject Classification. 55P99, 55P05

Key Words and Phrases. Coextension, Toda bracket.

*Professor Emeritus, Ibaraki University, Mito, Ibaraki 310-8512, Japan (hideaki.ooshima.mito@vc.ibaraki.ac.jp) 
Problem I. Does the equality $\operatorname{Coext}_{f}^{n}(g)=\operatorname{Coext}_{f}^{n}(g)^{*}$ hold?

While an extension is a natural notion, a coextension is not so natural. If the problem were always affirmative, we could obtain a new simpler definition of coextension. But the situation is not so convenient for us. Indeed we give two partial answers to Problem I. One is negative and the other is positive. A negative answer is

Example 1.1. Set $W=\mathrm{S}^{10}, X=\mathrm{S}^{4}, Y=\mathrm{S}^{3}, g=\nu_{4}^{2}$, and $f=\eta_{3}$ (for notations see [T2]). Then $f \circ g$ is null homotopic and there is a map $h: \Sigma^{5} W \rightarrow \Sigma^{4} Y \cup \Sigma_{\Sigma^{4}} C \Sigma^{4} X$ such that $q_{\Sigma^{4} f} \circ h$ is homotopic to $-\Sigma^{5} g$ and $h$ is not homotopic to an $n$-fold coextension of $\Sigma^{4-n} g$ w.r.t. $\Sigma^{4-n} f$ for each $n \in\{0,1,2,3,4\}$.

A positive answer is

Proposition 1.2. $\quad$ Suppose that $W, X, Y$ are $C W$-complexes having vertexes as base points and a map $h: \Sigma^{n+1} W \rightarrow \Sigma^{n} Y \cup_{\Sigma^{n} f} C \Sigma^{n} X$ satisfies that $q_{\Sigma^{n} f} \circ h$ is homotopic to $-\sum^{n+1} g$. If

$n+\min \left\{2 \cdot \operatorname{conn}(Y), 2 \cdot \operatorname{conn}\left(Y \cup_{f} C X\right)\right\}>\operatorname{dim} W \quad$ and $\quad 2 \cdot \operatorname{conn}(Y) \geq \operatorname{dim} W$,

then $h$ is homotopic to an $n$-fold coextension of $g$ w.r.t. $f$. If

$\operatorname{conn}(Y) \geq 0, \operatorname{conn}\left(Y \cup_{f} C X, Y\right) \geq 1, n+\operatorname{conn}\left(Y \cup_{f} C X, Y\right)+\operatorname{conn}(Y)>\operatorname{dim} W$, and the group $\left[\Sigma^{n+1} W, \Sigma^{n} Y \cup_{\Sigma^{n} f} C \Sigma^{n} X\right]$ is commutative,

then $h$ is homotopic to a coextension of $\Sigma^{n} g$, w.r.t. $\Sigma^{n} f$; if additionally $2 \cdot \operatorname{conn}(Y) \geq$ $\operatorname{dim} W$, then $h$ is homotopic to an $n$-fold coextension of $g$ w.r.t. $f$. Here conn denotes the connectivity.

For integers $m, k$ with $k \geq 2$, we set $m \iota_{k-1}=\Sigma^{k-2}\left(m \iota_{1}\right): \mathrm{S}^{k-1} \rightarrow \mathrm{S}^{k-1}$ and $M_{m}^{k}=\mathrm{S}^{k-1} \cup_{m \iota_{k-1}} C \mathrm{~S}^{k-1}$, where $\iota_{\ell}$ is the identity map of the $\ell$-dimensional sphere $\mathrm{S}^{\ell}$. We have

Corollary 1.3. The canonical homeomorphism $\varphi_{m}^{k}: \Sigma M_{m}^{k} \rightarrow M_{m}^{k+1}$ (for definition see Problem 6.1 below) is homotopic to a coextension of $q_{m \iota_{k-1}}: M_{m}^{k} \rightarrow \mathrm{S}^{k}$ w.r.t. $m \iota_{k}$ provided $k \geq 3$ or $k=m=2$.

Our second problem is the following for which we have no answer.

Problem II. Does the equality $\operatorname{Ext}_{\Sigma^{n} f}(e) \circ \operatorname{Coext}_{f}^{n}(g)=\operatorname{Ext}_{\Sigma^{n} f}(e) \circ \operatorname{Coext}_{f}^{n}(g)^{*}$ hold?

In $\S 2$, we recall from $[\mathbf{P}]$ a definition of a map between mapping cones. In $\S 3$, we study coextensions. We prove Example 1.1, Proposition 1.2, and Corollary 1.3 in $\S 4$, $\S 5$, and $\S 6$, respectively.

I would like to thank the referee for his/her suggestions from one of which (1.1) is changed to the present form.

\section{Preliminaries}


Throughout the paper, every topological space has the base point and every map between topological spaces is continuous and preserves base points. Let $I$ denote the unit interval $[0,1]$ with 1 as the base point. We denote the base point of spaces $X, Y, \ldots$ by $x_{0}, y_{0}, \ldots$ or simply $*$. The constant map $X \rightarrow Y, x \mapsto y_{0}$, is denoted by *. We set $X \wedge Y=(X \times Y) /\left(X \times\left\{y_{0}\right\} \cup\left\{x_{0}\right\} \times Y\right)$; the point of $X \wedge Y$ represented by $(x, y)$ is denoted by $x \wedge y$; the base point of $X \wedge Y$ is $x_{0} \wedge y_{0}$; the switching maps $X \times Y \rightarrow Y \times X,(x, y) \mapsto(y, x)$, and $X \wedge Y \rightarrow Y \wedge X, x \wedge y \mapsto y \wedge x$, are denoted by $\tau(X, Y)$. For a space $X$, let $1_{X}$ denote the identity map of $X$, and set $C X=X \wedge I$ and $\Sigma X=X \wedge(I /\{0,1\})$. The point of $\Sigma X$ represented by $(x, t) \in X \times I$ is denoted by $x \wedge \bar{t}$. Given maps $f, g: X \rightarrow Y$, if there is a map $H: X \times I \rightarrow Y$ such that $H\left(x_{0}, t\right)=y_{0}, H(x, 0)=f(x), H(x, 1)=g(x)(x \in X, t \in I)$, then we write $H: f \simeq g$ or $f \simeq g$ and we call $H$ a homotopy from $f$ to $g$; the relation $\simeq$ is an equivalence relation on the set of maps $X \rightarrow Y$ and the equivalence class of $f$ is called the homotopy class of $f$, and $[X, Y]$ denotes the set of homotopy classes of maps $X \rightarrow Y$. When $f \simeq *$, we say $f$ is null homotopic and the homotopy class of $f$ is denoted by 0 . A map $f: X \rightarrow Y$ is a homotopy equivalence if there is a map $g: Y \rightarrow X$ such that $g \circ f \simeq 1_{X}$ and $f \circ g \simeq 1_{Y}$. Given a map $f: X \rightarrow Y$, let $1_{f}: X \times I \rightarrow Y$ be defined by $1_{f}(x, t)=f(x), Y \cup_{f} C X$ the mapping cone of $f, i_{f}: Y \rightarrow Y \cup_{f} C X$ the inclusion map, and $q_{f}: Y \cup_{f} C X \rightarrow \Sigma X$ the map pinching $Y$ to a point. We denote by $\mathrm{S}^{n}$ the $n$-dimensional sphere, and we set $\Sigma^{n} X=X \wedge \mathrm{S}^{n}$ for any space $X$. We use identifications $I /\{0,1\}=\mathrm{S}^{1}$ and $\Sigma \mathrm{S}^{n}=\mathrm{S}^{n+1}$. For a homotopy $F: X \times I \rightarrow Y$, we define $\widetilde{\Sigma}^{n} F: \Sigma^{n} X \times I \rightarrow \Sigma^{n} Y$ by $\left(\widetilde{\Sigma}^{n} F\right)\left(x \wedge s_{n}, t\right)=F(x, t) \wedge s_{n}\left(x \in X, s_{n} \in \mathrm{S}^{n}, t \in I\right)$; if $F(X \times\{1\})=y_{0}$, that is, if $F: C X \rightarrow Y$, then $\widetilde{\Sigma}^{n} F=\left(F \wedge 1_{\mathrm{S}^{n}}\right) \circ\left(1_{X} \wedge \tau\left(\mathrm{S}^{n}, I\right)\right) \circ q$, where $q: \Sigma^{n} X \times I \rightarrow \Sigma^{n} X \wedge I=C \Sigma^{n} X$ is the quotient map, therefore, for the sake of economizing notations, we denote $\left(F \wedge 1_{\mathrm{S}^{n}}\right) \circ\left(1_{X} \wedge \tau\left(\mathrm{S}^{n}, I\right)\right)$ by $\widetilde{\Sigma}^{n} F$. Notice that $\Sigma^{0} X=X$ and $\widetilde{\Sigma}^{0} F=F$.

Definition 2.1. (cf. 2.2 of $[\mathbf{P}]$ ) Given a homotopy commutative square and a homotopy

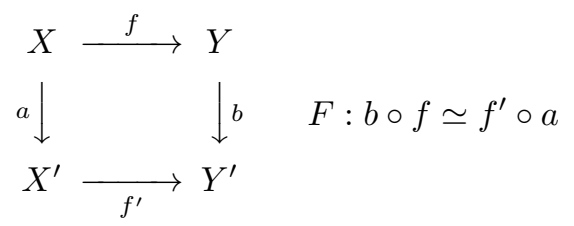

we define $\Phi\left(f, f^{\prime}, a, b ; F\right): Y \cup_{f} C X \rightarrow Y^{\prime} \cup_{f^{\prime}} C X^{\prime}$ by

$$
y \mapsto b(y), \quad x \wedge t \mapsto \begin{cases}F(x, 2 t) & 0 \leq t \leq 1 / 2 \\ a(x) \wedge(2 t-1) & 1 / 2 \leq t \leq 1\end{cases}
$$

\section{Lemma 2.2.}

(1) (Hilfssatz 7 of $[\mathbf{P} /)$ Suppose that (2.1) is given. Then the following diagram is 
homotopy commutative, while the middle square is strictly commutative.

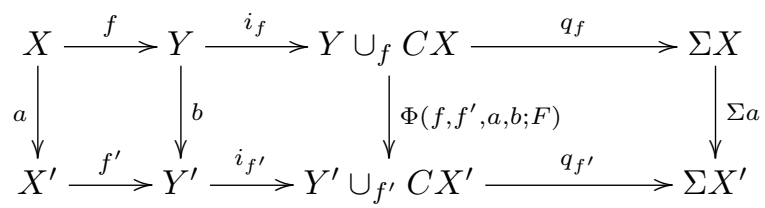

If $a, b$ are homotopy equivalences, then $\Phi\left(f, f^{\prime}, a, b ; F\right)$ is a homotopy equivalence.

(2) Let $F: f \simeq f^{\prime}: X \rightarrow Y$. Then

$$
\Phi\left(\Sigma^{n} f, \Sigma^{n} f^{\prime}, 1_{\Sigma^{n} X}, 1_{\Sigma^{n} Y} ; \widetilde{\Sigma}^{n} F\right): \Sigma^{n} Y \cup_{\Sigma^{n} f} C \Sigma^{n} X \rightarrow \Sigma^{n} Y \cup_{\Sigma^{n} f^{\prime}} C \Sigma^{n} X
$$

is a homotopy equivalence and its homotopy inverse is

$$
\Phi\left(\Sigma^{n} f^{\prime}, \Sigma^{n} f, 1_{\Sigma^{n} X}, 1_{\Sigma^{n} Y} ; \widetilde{\Sigma}^{n}(-F)\right): \Sigma^{n} Y \cup_{\Sigma^{n} f^{\prime}} C \Sigma^{n} X \rightarrow \Sigma^{n} Y \cup_{\Sigma^{n} f} C \Sigma^{n} X,
$$

where $-F: f^{\prime} \simeq f$ is the inverse homotopy of $F$, that is, $(-F)(x, t)=F(x, 1-t)$.

(3) For any map $f: X \rightarrow Y$, we have $\Phi\left(f, f, 1_{X}, 1_{Y} ; 1_{f}\right) \simeq 1_{Y \cup_{f} C X}$ rel $Y$.

Proof. For (1), see [P]. For (2), define a decomposition $I \times I=K_{1} \cup K_{2} \cup K_{3} \cup K_{4}$ and maps $u: I \times I \rightarrow I, \Psi:\left(\Sigma^{n} Y \cup_{\Sigma^{n} f} C \Sigma^{n} X\right) \times I \rightarrow \Sigma^{n} Y \cup_{\Sigma^{n} f} C \Sigma^{n} X$ by

$$
\begin{gathered}
K_{1}=\{(s, t) \in I \times I \mid t \leq-2 s+1\}, K_{2}=\left\{(s, t) \in I \times I \mid-2 s+1 \leq t \leq-\frac{4}{3} s+1\right\}, \\
K_{3}=\left\{(s, t) \in I \times I \mid-\frac{4}{3} s+1 \leq t \leq-s+1\right\}, \quad K_{4}=\{(s, t) \in I \times I \mid-s+1 \leq t\}, \\
u(s, t)= \begin{cases}2 s & (s, t) \in K_{1} \\
-4 s-3 t+3 & (s, t) \in K_{2} \\
4 s+3 t-3 & (s, t) \in K_{3} \\
s & (s, t) \in K_{4}\end{cases} \\
\Psi\left(y \wedge s_{n}, t\right)=y \wedge s_{n}, \Psi\left(x \wedge s_{n} \wedge s, t\right)=\left\{\begin{array}{ll}
F(x, u(s, t)) \wedge s_{n} & (s, t) \in K_{1} \cup K_{2} \\
x \wedge s_{n} \wedge u(s, t) & (s, t) \in K_{3} \cup K_{4}
\end{array} .\right.
\end{gathered}
$$

Then as is easily seen $\Psi$ is a homotopy from

$$
\Phi\left(\Sigma^{n} f^{\prime}, \Sigma^{n} f, 1_{\Sigma^{n} X}, 1_{\Sigma^{n} Y} ; \widetilde{\Sigma}^{n}(-F)\right) \circ \Phi\left(\Sigma^{n} f, \Sigma^{n} f^{\prime}, 1_{\Sigma^{n} X}, 1_{\Sigma^{n} Y} ; \widetilde{\Sigma}^{n} F\right)
$$

to $1_{\Sigma^{n} Y \cup_{\Sigma^{n} f} C \Sigma^{n} X}$ relative $\Sigma^{n} Y$. Since

$$
\Phi\left(\Sigma^{n} f, \Sigma^{n} f^{\prime}, 1_{\Sigma^{n} X}, 1_{\Sigma^{n} Y} ; \widetilde{\Sigma}^{n} F\right), \Phi\left(\Sigma^{n} f^{\prime}, \Sigma^{n} f, 1_{\Sigma^{n} X}, 1_{\Sigma^{n} Y} ; \widetilde{\Sigma}^{n}(-F)\right)
$$

are homotopy equivalences by (1), this proves (2).

For (3), we define $u: I \times I \rightarrow I$ and $H:\left(Y \cup_{f} C X\right) \times I \rightarrow Y \cup_{f} C X$ by

$$
u(s, t)= \begin{cases}0 & t \leq-2 s+1 \\ 2 s+t-1 & -2 s+1 \leq t \leq-s+1, \quad H(y, t)=y, H(x \wedge s, t)=x \wedge u(s, t) . \\ s & -s+1 \leq t\end{cases}
$$


Then $H: \Phi\left(f, f, 1_{X}, 1_{Y} ; 1_{f}\right) \simeq 1_{Y \cup_{f} C X}$ rel $Y$.

\section{Properties of coextensions}

Let maps $g: W \rightarrow X$ and $f: X \rightarrow Y$ be given. If a null homotopy $A: C W \rightarrow Y$ of $f \circ g$ exists, then $\widetilde{\Sigma}^{n} A: \Sigma^{n} f \circ \Sigma^{n} g \simeq *$.

Definition 3.1. A map $h: \Sigma W \rightarrow Y \cup_{f} C X$ is called a coextension of $g$ w.r.t. $f$ $[\mathbf{T 2}, \mathrm{p} .13]$ if

$$
h(w \wedge \bar{t})=\left\{\begin{array}{ll}
g(w) \wedge(1-2 t) & 0 \leq t \leq 1 / 2 \\
\in Y & 1 / 2 \leq t \leq 1
\end{array} \quad(w \in W, t \in I) .\right.
$$

If $A: C W \rightarrow Y$ is a null homotopy of $f \circ g$, then we define $\left(\Sigma^{n} f, \widetilde{\Sigma}^{n} A, \Sigma^{n} g\right): \Sigma \Sigma^{n} W \rightarrow$ $\Sigma^{n} Y \cup_{\Sigma^{n} f} C \Sigma^{n} X$ (cf. $\left.[\mathbf{O g}]\right)$ by

$$
\begin{aligned}
& \left(\Sigma^{n} f, \widetilde{\Sigma}^{n} A, \Sigma^{n} g\right)\left(w \wedge s_{n} \wedge \bar{t}\right)= \begin{cases}\left(\Sigma^{n} g\right)\left(w \wedge s_{n}\right) \wedge(1-2 t) & 0 \leq t \leq 1 / 2 \\
\left(\widetilde{\Sigma}^{n} A\right)\left(w \wedge s_{n} \wedge(2 t-1)\right) & 1 / 2 \leq t \leq 1\end{cases} \\
& =\left\{\begin{array}{ll}
g(w) \wedge s_{n} \wedge(1-2 t) & 0 \leq t \leq 1 / 2 \\
A(w \wedge(2 t-1)) \wedge s_{n} & 1 / 2 \leq t \leq 1
\end{array} \quad\left(w \in W, s_{n} \in \mathrm{S}^{n}, t \in I\right),\right.
\end{aligned}
$$

and we call it an $n$-fold coextension of $g$ w.r.t. $f$ because of the following equality

$$
\left(\Sigma^{n} f, \widetilde{\Sigma}^{n} A, \Sigma^{n} g\right)=\varphi_{f}^{n} \circ \Sigma^{n}(f, A, g) \circ\left(1_{W} \wedge \tau\left(\mathrm{S}^{n}, \mathrm{~S}^{1}\right)\right),
$$

where $\varphi_{f}^{n}: \Sigma^{n}\left(Y \cup_{f} C X\right) \rightarrow \Sigma^{n} Y \cup_{\Sigma^{n} f} C \Sigma^{n} X$ is the canonical homeomorphism (see Problem 6.1).

From the following lemma a 0 -fold coextension and a coextension are the same notion.

Lemma 3.2. $\quad$ There is a coextension of $g$ w.r.t. $f$ if and only if $f \circ g \simeq *$. Every coextension of $g$ w.r.t. $f$ has a form $(f, A, g)$ with $A: f \circ g \simeq *$.

Proof. Suppose that $h$ is a coextension of $g$ w.r.t. $f$. Define $A: C W \rightarrow Y$ by $A(w \wedge t)=h(w \wedge \overline{(t+1) / 2})$. Then $A(w \wedge 0)=h(w \wedge \overline{1 / 2})=g(w) \wedge 0=f \circ g(w)$ and $A(w \wedge 1)=*$ so that $A$ is a null homotopy $f \circ g \simeq *$ and $h=(f, A, g)$.

Conversely suppose that $A: f \circ g \simeq *$ is given. Then, as is easily seen, $(f, A, g)$ is a coextension of $g$ w.r.t. $f$.

Lemma 3.3. (cf. $\S 3$ of $[\mathrm{Og}])$ Given homotopies $A: f \circ g \simeq *, F: f \simeq f^{\prime}$, and $G: g \simeq g^{\prime}$, we define $A^{F, G}: W \times I \rightarrow Y$ by

$$
\begin{gathered}
A^{F, G}(w, s)=\left\{\begin{array}{ll}
F(G(w, 1-2 s), 1-2 s) & 0 \leq s \leq 1 / 2 \\
A(w, 2 s-1) & 1 / 2 \leq s \leq 1
\end{array} . \text { Then } A^{F, G}: f^{\prime} \circ g^{\prime} \simeq *\right. \\
\widetilde{\Sigma}^{n} A^{F, G}=\left(\widetilde{\Sigma}^{n} A\right)^{\widetilde{\Sigma}^{n} F, \widetilde{\Sigma}^{n} G, \text { and }} \\
\Phi\left(\Sigma^{n} f, \Sigma^{n} f^{\prime}, 1_{\Sigma^{n} X}, 1_{\Sigma^{n} Y} ; \widetilde{\Sigma}^{n} F\right) \circ\left(\Sigma^{n} f, \widetilde{\Sigma}^{n} A, \Sigma^{n} g\right) \simeq\left(\Sigma^{n} f^{\prime}, \widetilde{\Sigma}^{n} A^{F, G}, \Sigma^{n} g^{\prime}\right), \\
\left(\Sigma^{n} f, \widetilde{\Sigma}^{n} A, \Sigma^{n} g\right) \simeq\left(\Sigma^{n} f, \widetilde{\Sigma}^{n} A^{1_{f}, G}, \Sigma^{n} g^{\prime}\right) .
\end{gathered}
$$


Proof. As is easily seen, $A^{F, G}: f^{\prime} \circ g^{\prime} \simeq *$ and $\left(\widetilde{\Sigma}^{n} A\right)^{\widetilde{\Sigma}^{n} F, \widetilde{\Sigma}^{n} G}=\widetilde{\Sigma}^{n} A^{F, G}$. We divide $I \times I=K_{1} \cup \cdots \cup K_{5}$ and define $u: K_{1} \cup K_{2} \rightarrow I, v: K_{3} \cup K_{4} \cup K_{5} \rightarrow I$, and $\Psi: \Sigma \Sigma^{n} W \times I \rightarrow \Sigma^{n} Y \cup_{\Sigma^{n} f^{\prime}} C \Sigma^{n} X$ as follows:

$$
\begin{gathered}
K_{1}=\{(s, t) \in I \times I \mid 2 s \leq t\}, \quad K_{2}=\{(s, t) \in I \times I \mid 4 s-1 \leq t \leq 2 s\}, \\
K_{3}=\{(s, t) \in I \times I \mid 4 s-2 \leq t \leq 4 s-1\}, \quad K_{4}=\{(s, t) \in I \times I \mid 2 s-1 \leq t \leq 4 s-2\}, \\
K_{5}=\{(s, t) \in I \times I \mid t \leq 2 s-1\}, \\
u(s, t)=\left\{\begin{array}{ll}
-2 s+1 \quad(s, t) \in K_{1} \\
-4 s+t+1 \quad & (s, t) \in K_{2}
\end{array}, \quad v(s, t)= \begin{cases}-4 s+t+2 & (s, t) \in K_{3} \\
4 s-t-2 & (s, t) \in K_{4}, \\
2 s-1 & (s, t) \in K_{5}\end{cases} \right. \\
\Psi\left(w \wedge s_{n} \wedge \bar{s}, t\right)= \begin{cases}G(w, t) \wedge s_{n} \wedge u(s, t) & (s, t) \in K_{1} \cup K_{2} \\
F(G(w, t \cdot v(s, t)), v(s, t)) \wedge s_{n} & (s, t) \in K_{3} \\
A(w, v(s, t)) \wedge s_{n} & (s, t) \in K_{4} \cup K_{5}\end{cases} \\
\left(w \in W, s_{n} \in \mathrm{S}^{n}, s, t \in I\right) .
\end{gathered}
$$

Then $\Psi: \Phi\left(\Sigma^{n} f, \Sigma^{n} f^{\prime}, 1_{\Sigma^{n} X}, 1_{\Sigma^{n} Y} ; \widetilde{\Sigma}^{n} F\right) \circ\left(\Sigma^{n} f, \widetilde{\Sigma}^{n} A, \Sigma^{n} g\right) \simeq\left(\Sigma^{n} f^{\prime}, \widetilde{\Sigma}^{n} A^{F, G}, \Sigma^{n} g^{\prime}\right)$. This proves (3.1). By Lemma 2.2(3) and (3.1), we have (3.2).

Definition 3.4. We denote the set of homotopy classes of $n$-fold coextensions of $g$ w.r.t. $f$ by $\operatorname{Coext}_{f}^{n}(g)$ which is a subset of $\left[\Sigma \Sigma^{n} W, \Sigma^{n} Y \cup_{\Sigma^{n} f} C \Sigma^{n} X\right]$.

\section{Lemma 3.5.}

(1) $\operatorname{Coext}_{f}^{n}(g) \subset \operatorname{Coext}_{\Sigma f}^{n-1}(\Sigma g)$ for $n \geq 1$.

(2) (cf. $\left[\mathrm{Og}\right.$, Lemmas (3.2) and (3.3)]) Let $F: f \simeq f^{\prime}, G: g \simeq g^{\prime}$. Then $\Phi\left(\Sigma^{n} f, \Sigma^{n} f^{\prime}, 1_{\Sigma^{n} X}, 1_{\Sigma^{n} Y} ; \widetilde{\Sigma}^{n} F\right) \circ \operatorname{Coext}_{f}^{n}(g)=\operatorname{Coext}_{f^{\prime}}^{n}\left(g^{\prime}\right), \operatorname{Coext}_{f}^{n}(g)=\operatorname{Coext}_{f}^{n}\left(g^{\prime}\right)$.

Proof. (1) Let $A: f \circ g \simeq *$ and $n \geq 1$. Then $\widetilde{\Sigma} A: \Sigma f \circ \Sigma g \simeq *$ and $\widetilde{\Sigma}^{n} A=\widetilde{\Sigma}^{n-1} \widetilde{\Sigma} A$ so that $\left(\Sigma^{n} f, \widetilde{\Sigma}^{n} A, \Sigma^{n} g\right)=\left(\Sigma^{n-1} \Sigma f, \widetilde{\Sigma}^{n-1} \widetilde{\Sigma} A, \Sigma^{n-1} \Sigma g\right)$. Hence (1) is proved.

(2) We use the situations of Lemma 3.3. By Lemmas 2.2(2) and 3.3, we have

$\operatorname{Coext}_{f^{\prime}}^{n}\left(g^{\prime}\right)$

$$
\begin{aligned}
& =\Phi\left(\Sigma^{n} f, \Sigma^{n} f^{\prime}, 1_{\Sigma^{n} X}, 1_{\Sigma^{n} Y} ; \widetilde{\Sigma}^{n} F\right) \circ \Phi\left(\Sigma^{n} f^{\prime}, \Sigma^{n} f, 1_{\Sigma^{n} X}, 1_{\Sigma^{n} Y} ; \widetilde{\Sigma}^{n}(-F)\right) \circ \operatorname{Coext}_{f^{\prime}}^{n}\left(g^{\prime}\right) \\
& \subset \Phi\left(\Sigma^{n} f, \Sigma^{n} f^{\prime}, 1_{\Sigma^{n} X}, 1_{\Sigma^{n} Y} ; \widetilde{\Sigma}^{n} F\right) \circ \operatorname{Coxt}_{f}^{n}(g) \subset \operatorname{Coext}_{f^{\prime}}^{n}\left(g^{\prime}\right)
\end{aligned}
$$

so that the last containment is equality. Take $f^{\prime}=f$ and $F=1_{f}$. Since $\widetilde{\Sigma}^{n} 1_{f}=1_{\Sigma^{n} f}$, we have the last equality in (2) by Lemma $2.2(3)$.

By Lemma 3.5(2), we can define

Definition 3.6. Let $\gamma \in[W, X]$ be the homotopy class of $g$. We set $\operatorname{Coext}_{f}^{n}(\gamma)=$ $\operatorname{Coext}_{f}^{n}(g)$, and we call an element of $\operatorname{Coext}_{f}^{n}(\gamma)$ an $n$-fold coextension of $\gamma$ w.r.t. $f$. 


\section{Proposition 3.7.}

(1) $\operatorname{Coext}_{f}^{n}(\gamma)$ is not empty if and only if $f \circ g \simeq *$.

(2) If $\widetilde{\gamma} \in \operatorname{Coext}_{f}^{n}(\gamma)$, then

$$
\begin{gathered}
\operatorname{Coext}_{f}^{n}(\gamma)=\widetilde{\gamma}+i_{\Sigma^{n} f} \circ \Sigma^{n}[\Sigma W, Y] \circ\left(1_{W} \wedge \tau\left(\mathrm{S}^{n}, \mathrm{~S}^{1}\right)\right) \\
\operatorname{Coext}_{\Sigma^{n} f}\left(\Sigma^{n} \gamma\right)=\widetilde{\gamma}+i_{\Sigma^{n} f} \circ\left[\Sigma \Sigma^{n} W, \Sigma^{n} Y\right](c f .[\mathbf{O g}, \text { Proposition (3.4) }))
\end{gathered}
$$

Proof. (1) is obvious from definitions.

Let us prove (2). Let $\left(\Sigma^{n} f, \widetilde{\Sigma}^{n} A, \Sigma^{n} g\right)$ represent $\widetilde{\gamma} \in \operatorname{Coext}_{f}^{n}(\gamma)$.

First take $\widehat{\gamma} \in \operatorname{Coext}_{f}^{n}(\gamma)$. Let $\left(\Sigma^{n} f, \widetilde{\Sigma}^{n} B, \Sigma^{n} g\right)$ represent $\widehat{\gamma}$, where $B: f \circ g \simeq *$. We define $m: \Sigma W \rightarrow Y$ by

$$
m(w \wedge \bar{s})=\left\{\begin{array}{ll}
A(w \wedge(1-2 s)) & 0 \leq s \leq 1 / 2 \\
B(w \wedge(2 s-1)) & 1 / 2 \leq s \leq 1
\end{array}(w \in W, s \in I)\right.
$$

We are going to show $\left(\Sigma^{n} f, \widetilde{\Sigma}^{n} B, \Sigma^{n} g\right) \simeq\left(\Sigma^{n} f, \widetilde{\Sigma}^{n} A, \Sigma^{n} g\right)+i_{\Sigma^{n} f} \circ \Sigma^{n} m \circ\left(1_{W} \wedge\right.$ $\left.\tau\left(\mathrm{S}^{n}, \mathrm{~S}^{1}\right)\right)$. We have

$$
\left(\Sigma^{n} f, \widetilde{\Sigma}^{n} B, \Sigma^{n} g\right)\left(w \wedge s_{n} \wedge \bar{s}\right)= \begin{cases}g(w) \wedge s_{n} \wedge(1-2 s) & 0 \leq s \leq 1 / 2 \\ B(w \wedge(2 s-1)) \wedge s_{n} & 1 / 2 \leq s \leq 1\end{cases}
$$

and

$$
\begin{aligned}
\left(\left(\Sigma^{n} f, \widetilde{\Sigma}^{n} A, \Sigma^{n} g\right)+\right. & \left.i_{\Sigma^{n} f} \circ \Sigma^{n} m \circ\left(1_{W} \wedge \tau\left(\mathrm{S}^{n}, \mathrm{~S}^{1}\right)\right)\right)\left(w \wedge s_{n} \wedge \bar{s}\right) \\
= & \begin{cases}g(w) \wedge s_{n} \wedge(1-4 s) & 0 \leq s \leq 1 / 4 \\
A(w \wedge(4 s-1)) \wedge s_{n} & 1 / 4 \leq s \leq 1 / 2 \\
A(w \wedge(3-4 s)) \wedge s_{n} & 1 / 2 \leq s \leq 3 / 4 \\
B(w \wedge(4 s-3)) \wedge s_{n} & 3 / 4 \leq s \leq 1\end{cases}
\end{aligned}
$$

We divide $I \times I=K_{1} \cup \cdots \cup K_{6}$ as follows.

$$
\begin{gathered}
K_{1}=\{(s, t) \in I \times I \mid t \leq-2 s+1\}, \quad K_{2}=\{(s, t) \in I \times I \mid-2 s+1 \leq t \leq-4 t+2\}, \\
K_{3}=\{(s, t) \in I \times I \mid-4 s+2 \leq t \text { and } s \leq 1 / 2\} \\
K_{4}=\{(s, t) \in I \times I \mid 1 / 2 \leq s \text { and } 4 s-2 \leq t\} \\
K_{5}=\{(s, t) \in I \times I \mid 2 s-1 \leq t \leq 4 s-2\}, \quad K_{6}=\{(s, t) \in I \times I \mid t \leq 2 s-1\} .
\end{gathered}
$$


We define $u: I \times I \rightarrow I$ and $\Phi: \Sigma \Sigma^{n} W \times I \rightarrow \Sigma^{n} Y \cup_{\Sigma^{n} f} C \Sigma^{n} X$ by

$$
\begin{gathered}
u(s, t)= \begin{cases}-2 s+1 & (s, t) \in K_{1} \\
-4 s-t+2 & (s, t) \in K_{2} \\
4 s+t-2 & (s, t) \in K_{3} \\
-4 s+t+2 & (s, t) \in K_{4} \\
4 s-t-2 & (s, t) \in K_{5} \\
2 s-1 & (s, t) \in K_{6}\end{cases} \\
\Phi\left(w \wedge s_{n} \wedge \bar{s}, t\right)= \begin{cases}g(w) \wedge s_{n} \wedge u(s, t) & (s, t) \in K_{1} \cup K_{2} \\
A(w \wedge u(s, t)) \wedge s_{n} & (s, t) \in K_{3} \cup K_{4} . \\
B(w \wedge u(s, t)) \wedge s_{n} & (s, t) \in K_{5} \cup K_{6}\end{cases}
\end{gathered}
$$

Then $\Phi:\left(\Sigma^{n} f, \widetilde{\Sigma}^{n} B, \Sigma^{n} g\right) \simeq\left(\Sigma^{n} f, \widetilde{\Sigma}^{n} A, \Sigma^{n} g\right)+i_{\Sigma^{n} f} \circ \Sigma^{n} m \circ\left(1_{W} \wedge \tau\left(\mathrm{S}^{n}, \mathrm{~S}^{1}\right)\right)$. Thus $\widehat{\gamma} \in \widetilde{\gamma}+i_{\Sigma^{n} f} \circ \Sigma^{n}[\Sigma W, Y] \circ\left(1_{W} \wedge \tau\left(\mathrm{S}^{n}, \mathrm{~S}^{1}\right)\right)$ and so

$$
\operatorname{Coext}_{f}^{n}(\gamma) \subset \widetilde{\gamma}+i_{\Sigma^{n} f} \circ \Sigma^{n}[\Sigma W, Y] \circ\left(1_{W} \wedge \tau\left(\mathrm{S}^{n}, \mathrm{~S}^{1}\right)\right) .
$$

Secondly let $m \in \mu \in[\Sigma W, Y]$. Then $\left(\Sigma^{n} f, \widetilde{\Sigma}^{n} A, \Sigma^{n} g\right)+i_{\Sigma^{n} f} \circ \Sigma^{n} m \circ\left(1_{W} \wedge\right.$ $\left.\tau\left(\mathrm{S}^{n}, \mathrm{~S}^{1}\right)\right)$ represents $\widetilde{\gamma}+i_{\Sigma^{n} f} \circ \Sigma^{n} \mu \circ\left(1_{W} \wedge \tau\left(\mathrm{S}^{n}, \mathrm{~S}^{1}\right)\right)$. We define $B: C W \rightarrow Y$ by

$$
B(w \wedge s)=\left\{\begin{array}{ll}
A(w \wedge(2 s)) & 0 \leq s \leq 1 / 2 \\
m(w \wedge \overline{2 s-1}) & 1 / 2 \leq s \leq 1
\end{array} .\right.
$$

Then $B: f \circ g \simeq *$. For $w \in W, s_{n} \in \mathrm{S}^{n}, s \in I$, we have

$$
\left(\Sigma^{n} f, \widetilde{\Sigma}^{n} B, \Sigma^{n} g\right)\left(w \wedge s_{n} \wedge \bar{s}\right)= \begin{cases}g(w) \wedge s_{n} \wedge(1-2 s) & 0 \leq s \leq 1 / 2 \\ A(w \wedge(4 s-2)) \wedge s_{n} & 1 / 2 \leq s \leq 3 / 4 \\ m(w \wedge \overline{4 s-3}) \wedge s_{n} & 3 / 4 \leq s \leq 1\end{cases}
$$

and

$$
\begin{aligned}
\left(\left(\Sigma^{n} f, \widetilde{\Sigma}^{n} A, \Sigma^{n} g\right)+\right. & \left.i_{\Sigma^{n} f} \circ \Sigma^{n} m \circ\left(1_{W} \wedge \tau\left(\mathrm{S}^{n}, \mathrm{~S}^{1}\right)\right)\right)\left(w \wedge s_{n} \wedge \bar{s}\right) \\
= & \begin{cases}g(w) \wedge s_{n} \wedge(1-4 s) & 0 \leq s \leq 1 / 4 \\
A(w \wedge(4 s-1)) \wedge s_{n} & 1 / 4 \leq s \leq 1 / 2 . \\
m(w \wedge \overline{2 s-1}) \wedge s_{n} & 1 / 2 \leq s \leq 1\end{cases}
\end{aligned}
$$

We divide $I \times I=K_{1} \cup \cdots \cup K_{5}$ as follows.

$$
\begin{gathered}
K_{1}=\{(s, t) \in I \times I \mid t \leq-2 s+1\}, K_{2}=\{(s, t) \in I \times I \mid-2 s+1 \leq t \leq-4 s+2\}, \\
K_{3}=\{(s, t) \in I \times I \mid-4 s+2 \leq t \leq-4 s+3\}, \\
K_{4}=\{(s, t) \in I \times I \mid-4 s+3 \leq t \leq-2 s+2\}, K_{5}=\{(s, t) \in I \times I \mid-2 s+2 \leq t\} .
\end{gathered}
$$

We define $u: K_{1} \cup K_{2} \cup K_{3} \rightarrow I$ and $v: K_{4} \cup K_{5} \rightarrow I$ by

$$
u(s, t)=\left\{\begin{array}{ll}
-2 s+1 & (s, t) \in K_{1} \\
-4 s-t+2 & (s, t) \in K_{2}, \\
4 s+t-2 & (s, t) \in K_{3}
\end{array} \quad v(s, t)=\left\{\begin{array}{ll}
4 s+t-3 & (s, t) \in K_{4} \\
2 s-1 & (s, t) \in K_{5}
\end{array} .\right.\right.
$$


We define $\Phi: \Sigma \Sigma^{n} W \times I \rightarrow \Sigma^{n} Y \cup_{\Sigma^{n} f} C \Sigma^{n} X$ by

$$
\Phi\left(w \wedge s_{n} \wedge \bar{s}, t\right)=\left\{\begin{array}{ll}
g(w) \wedge s_{n} \wedge u(s, t) & (s, t) \in K_{1} \cup K_{2} \\
A(w \wedge u(s, t)) \wedge s_{n} & (s, t) \in K_{3} \\
m(w \wedge \overline{v(s, t)}) \wedge s_{n} & (s, t) \in K_{4} \cup K_{5}
\end{array} .\right.
$$

Then $\Phi:\left(\Sigma^{n} f, \widetilde{\Sigma}^{n} B, \Sigma^{n} g\right) \simeq\left(\Sigma^{n} f, \widetilde{\Sigma}^{n} A, \Sigma^{n} g\right)+i_{\Sigma^{n} f} \circ \Sigma^{n} m \circ\left(1_{W} \wedge \tau\left(\mathrm{S}^{n}, \mathrm{~S}^{1}\right)\right)$. This means the homotopy class of $\left(\Sigma^{n} f, \widetilde{\Sigma}^{n} A, \Sigma^{n} g\right)+i_{\Sigma^{n} f} \circ \Sigma^{n} m \circ\left(1_{W} \wedge \tau\left(\mathrm{S}^{n}, \mathrm{~S}^{1}\right)\right)$ is in $\operatorname{Coext}_{f}^{n}(\gamma)$. Hence $\widetilde{\gamma}+i_{\Sigma^{n} f} \circ \Sigma^{n}[\Sigma W, Y] \circ\left(1_{W} \wedge \tau\left(\mathrm{S}^{n}, \mathrm{~S}^{1}\right)\right) \subset \operatorname{Coext}_{f}^{n}(\gamma)$. Therefore

$$
\operatorname{Coext}_{f}^{n}(\gamma)=\widetilde{\gamma}+i_{\Sigma^{n} f} \circ \Sigma^{n}[\Sigma W, Y] \circ\left(1_{W} \wedge \tau\left(\mathrm{S}^{n}, \mathrm{~S}^{1}\right)\right) .
$$

Replace $\Sigma^{n} W, \Sigma^{n} X, \Sigma^{n} Y, \Sigma^{n} \gamma, \Sigma^{n} f$ with $W^{\prime}, X^{\prime}, Y^{\prime}, \gamma^{\prime}, f^{\prime}$, respectively, and take $n^{\prime}=$ 0 . Then we have $\operatorname{Coext}_{f^{\prime}}\left(\gamma^{\prime}\right)=\widetilde{\gamma}+i_{f^{\prime}} \circ\left[\Sigma W^{\prime}, Y^{\prime}\right]$, that is, $\operatorname{Coext}_{\Sigma^{n} f}\left(\Sigma^{n} \gamma\right)=\widetilde{\gamma}+$ $i_{\Sigma^{n} f} \circ\left[\Sigma \Sigma^{n} W, \Sigma^{n} Y\right]$. This completes the proof of $(2)$.

Consider the sequence:

$$
\left[\Sigma^{n+1} W, \Sigma^{n} Y\right] \stackrel{i_{\Sigma^{n} f_{*}}}{\longrightarrow}\left[\Sigma^{n+1} W, \Sigma^{n} Y \cup_{\Sigma^{n} f} C \Sigma^{n} X\right] \stackrel{q_{\Sigma^{n} f_{*}}}{\longrightarrow}\left[\Sigma^{n+1} W, \Sigma^{n+1} X\right]
$$

Obviously Image $\left(i_{\Sigma^{n} f_{*}}\right) \subset \operatorname{Kernel}\left(q_{\Sigma^{n} f_{*}}\right)$. We have

Corollary 3.8. Suppose $f \circ g \simeq *$.

(1) If Image $\left(i_{\Sigma^{n} f_{*}}\right) \varsubsetneqq \operatorname{Kernel}\left(q_{\Sigma^{n} f_{*}}\right)$, then Problem 1.1 is negative.

(2) If Image $\left(i_{\Sigma^{n} f_{*}}\right)=\operatorname{Kernel}\left(q_{\Sigma^{n} f_{*}}\right)$ and the group $\left[\Sigma^{n+1} W, \Sigma^{n} Y \cup_{\Sigma^{n} f} C \Sigma^{n} X\right]$ is commutative, then any $\widehat{\gamma} \in\left[\Sigma^{n+1} W, \Sigma^{n} Y \cup_{\Sigma^{n} f} C \Sigma^{n} X\right]$ with $q_{\Sigma^{n} f_{*}} \widehat{\gamma}=-\Sigma^{n+1} \gamma$ is in $\operatorname{Coext}_{\Sigma^{n} f}\left(\Sigma^{n} \gamma\right)$. If additionally $W, Y$ are $C W$-complexes with vertexes as base points and $2 \cdot \operatorname{conn}(Y) \geq \operatorname{dim} W$, then $\widehat{\gamma} \in \operatorname{Coext}_{f}^{n}(\gamma)$, that is, Problem 1.1 is positive.

Proof. Take $\widetilde{\gamma} \in \operatorname{Coext}_{f}^{n}(\gamma)$.

(1) Suppose Image $\left(i_{\Sigma^{n} f_{*}}\right) \varsubsetneqq \operatorname{Kernel}\left(q_{\Sigma^{n} f_{*}}\right)$. Take $\alpha \in \operatorname{Kernel}\left(q_{\Sigma^{n} f_{*}}\right)$ which is not in Image $\left(i_{\Sigma^{n} f_{*}}\right)$, and set $\widehat{\gamma}=\widetilde{\gamma}+\alpha$. Then $q_{\Sigma^{n} f_{*}}(\widehat{\gamma})=-\Sigma^{n+1} \gamma$ and $\widehat{\gamma}$ is not in $\operatorname{Coext}_{f}^{n}(\gamma)$ by Proposition 3.7(2). Hence Problem 1.1 is negative.

(2) Suppose Image $\left(i_{\Sigma^{n} f_{*}}\right)=\operatorname{Kernel}\left(q_{\Sigma^{n} f_{*}}\right)$ and the group $\left[\Sigma^{n+1} W, \Sigma^{n} Y \cup_{\Sigma^{n} f}\right.$ $\left.C \Sigma^{n} X\right]$ is commutative. Let $\widehat{\gamma} \in\left[\Sigma^{n+1} W, \Sigma^{n} Y \cup_{\Sigma^{n} f} C \Sigma^{n} X\right]$ with $q_{\Sigma^{n}} f_{*}(\widehat{\gamma})=-\Sigma^{n+1} \gamma$. Then $\widehat{\gamma}-\widetilde{\gamma} \in \operatorname{Kernel}\left(q_{\Sigma^{n} f_{*}}\right)$. Hence there exists $\mu \in\left[\Sigma^{n+1} W, \Sigma^{n} Y\right]$ such that $\widehat{\gamma}=$ $i_{\Sigma^{n} f_{*}}(\mu)+\widetilde{\gamma}$. Since the group $\left[\Sigma^{n+1} W, \Sigma^{n} Y \cup_{\Sigma^{n} f} C \Sigma^{n} X\right]$ is commutative by the assumption, we have $\widehat{\gamma}=\widetilde{\gamma}+i_{\Sigma^{n} f_{*}}(\mu)$ and so $\widehat{\gamma} \in \operatorname{Coext}_{\Sigma^{n} f}\left(\Sigma^{n} \gamma\right)$ by Proposition 3.7(2). If additionally $W, Y$ are CW-complexes and $2 \cdot \operatorname{conn}(Y) \geq \operatorname{dim} W$, then $\Sigma^{n}$ : $[\Sigma W, Y] \rightarrow\left[\Sigma^{n+1} W, \Sigma^{n} Y\right]$ is surjective so that $\widehat{\gamma} \in \operatorname{Coext}_{f}^{n}(\gamma)$ by Proposition 3.7(2). This completes the proof.

\section{Proof of Example 1.1}


In this section we do not distinguish in notation between a map and its homotopy class.

Consider the following commutative diagram.

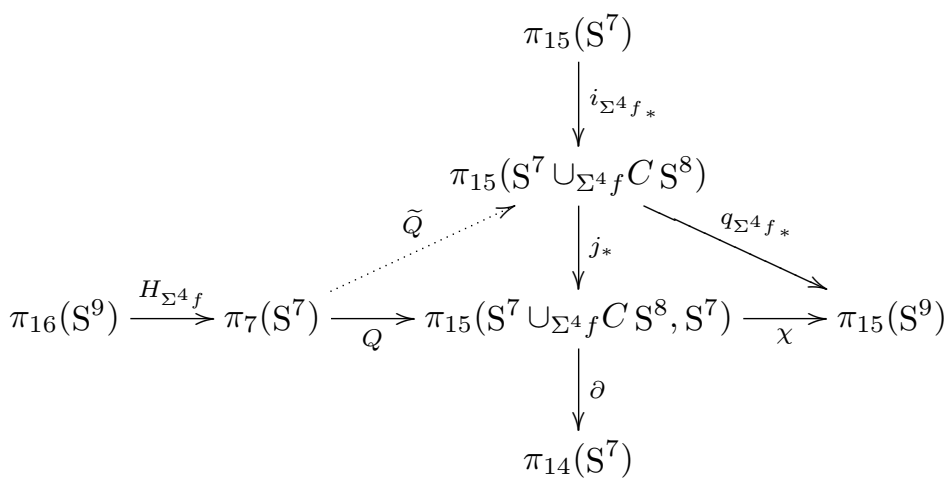

Here the vertical sequence is the homotopy exact sequence of the pair $\left(\mathrm{S}^{7} \cup \Sigma_{\Sigma_{f}} C \mathrm{~S}^{8}, \mathrm{~S}^{7}\right)$ and the horizontal sequence is the exact sequence in [J, Theorem $(2.1)]$, where $Q(x)$ is the relative Whitehead product $[\mathbf{T} \mathbf{1}]$ of $x \in \pi_{7}\left(\mathrm{~S}^{7}\right)$ and a generator $\varphi$ of $\pi_{9}\left(\mathrm{~S}^{7} \cup_{\Sigma^{4} f} C \mathrm{~S}^{8}\right.$, $\left.\mathrm{S}^{7}\right) \cong \mathbb{Z}, \chi$ is induced by shrinking $\mathrm{S}^{7}$ to a point, and $j:\left(\mathrm{S}^{7} \cup_{\Sigma^{4} f} C \mathrm{~S}^{8}, *\right) \rightarrow$ $\left(\mathrm{S}^{7} \cup_{\Sigma^{4} f} C \mathrm{~S}^{8}, \mathrm{~S}^{7}\right)$ is the inclusion map. By the definition of $H_{\Sigma^{4} f}$ in $[\mathbf{J}, \mathrm{p} .262]$, $H_{\Sigma^{4} f}=0$. Hence $Q$ is injective. Recall from $[\mathbf{T 1},(2.18)]$ that $\partial \circ Q(x)$ is the Whitehead product of $x$ and $\partial \varphi$ so that $\partial \circ Q(x)=0$, since $\mathrm{S}^{7}$ is an H-space. Hence there is a homomorphism $\widetilde{Q}: \pi_{7}\left(\mathrm{~S}^{7}\right) \rightarrow \pi_{15}\left(\mathrm{~S}^{7} \cup_{\Sigma^{4} f} C \mathrm{~S}^{8}\right)$ such that $j_{*} \circ \widetilde{Q}=Q$. Let $\widetilde{g}: \mathrm{S}^{15} \rightarrow \mathrm{S}^{7} \cup_{\Sigma^{4} f} C \mathrm{~S}^{8}$ be a coextension of $\Sigma^{4} g$ w.r.t. $\Sigma^{4} f$. Set $h_{k}=\widetilde{g}+\widetilde{Q}\left(k \iota_{7}\right)$ for each non zero integer $k$. Then $q_{\Sigma^{4} f} \circ h_{k}=q_{\Sigma^{4} f} \circ \widetilde{g}=-\Sigma^{5} g$. Since $\widetilde{Q}\left(k \iota_{7}\right)$ $(k \neq 0)$ is of infinite order and $\pi_{15}\left(\mathrm{~S}^{7}\right)$ is a finite group, it follows that $h_{k}$ is not in $\widetilde{g}+i_{\Sigma^{4} f} \circ \pi_{15}\left(\mathrm{~S}^{7}\right)$. Therefore $h_{k}$ is not in $\operatorname{Coext}_{\Sigma^{4} f}\left(\Sigma^{4} g\right)$ by Proposition 3.7(2). Since $\operatorname{Coext}_{f}^{4}(g) \subset \operatorname{Coext}_{\Sigma f}^{3}(\Sigma g) \subset \operatorname{Coext}_{\Sigma^{2} f}^{2}\left(\Sigma^{2} g\right) \subset \operatorname{Coext}_{\Sigma^{3} f}^{1}\left(\Sigma^{3} g\right) \subset \operatorname{Coext}_{\Sigma^{4} f}\left(\Sigma^{4} g\right)$ by Lemma 3.5(1), this proves Example 1.1.

\section{Proof of Proposition 1.2}

By Lemma 2.2(1) and (3.1), without loosing generalities, we can assume that $f$ is cellular so that $\Sigma^{n} Y \cup_{\Sigma^{n} f} C \Sigma^{n} X$ is a CW-complex. Let $\gamma \in[W, X]$ be the homotopy class of $g$. Let $\widetilde{\gamma} \in\left[\Sigma \Sigma^{n} W, \Sigma^{n} Y \cup_{\Sigma^{n} f} C \Sigma^{n} X\right]$ be an $n$-fold coextension of $\gamma$ w.r.t. $f$ (it exists because $f \circ g \simeq *)$. Let $\widehat{\gamma} \in\left[\Sigma \Sigma^{n} W, \Sigma^{n} Y \cup_{\Sigma^{n} f} C \Sigma^{n} X\right]$ with $q_{\Sigma^{n} f_{*}} \widehat{\gamma}=-\Sigma^{n+1} \gamma$ be given.

First assume (1.1). For any spaces $K, L$, we set $\{K, L\}=\operatorname{colimit}\left[\Sigma^{m} K, \Sigma^{m} L\right]$ and $\Sigma^{\infty}:[K, L] \rightarrow\{K, L\}$ denotes the stabilization. We have $q_{f_{*}}\left(\Sigma^{\infty}(\widehat{\gamma}-\widetilde{\gamma})\right)=0$. Since any cofibration is a fibration in the stable category (cf. [tD, p.184]), there exists $\mu \in\left\{\Sigma \Sigma^{n} W, \Sigma^{n} Y\right\}$ such that $\Sigma^{\infty}(\widehat{\gamma}-\widetilde{\gamma})=i_{\Sigma^{n} f_{*}} \mu$. Under the assumptions, we have $\Sigma^{\infty}:\left[\Sigma \Sigma^{n} W, \Sigma^{n} Y\right] \cong\left\{\Sigma \Sigma^{n} W, \Sigma^{n} Y\right\}$ and $\Sigma^{*}:\left[\Sigma \Sigma^{n} W, \Sigma^{n} Y \cup_{\Sigma^{n} f} C \Sigma^{n} X\right] \cong$ $\left\{\Sigma \Sigma^{n} W, \Sigma^{n} Y \cup_{\Sigma^{n} f} C \Sigma^{n} X\right\}$. Hence we may take $\mu \in\left[\Sigma \Sigma^{n} W, \Sigma^{n} Y\right]$. Then $\widehat{\gamma}-\widetilde{\gamma}=$ $i_{\Sigma^{n} f} \circ \mu$ so that $\widehat{\gamma}=i_{\Sigma^{n} f} \circ \mu+\widetilde{\gamma}=\widetilde{\gamma}+i_{\Sigma^{n} f} \circ \mu$. Since $\Sigma^{n}:[\Sigma W, Y] \rightarrow\left[\Sigma^{n+1} W, \Sigma^{n} Y\right]$ is 
surjective by the assumption $2 \cdot \operatorname{conn}(Y) \geq \operatorname{dim} W$, therefore $\widehat{\gamma}$ is an $n$-fold coextension of $\gamma$ w.r.t. $f$ by Proposition 3.7(2).

Secondly assume (1.2). By Corollary 3.8, it is worth proving that the sequence (5.1) below is exact under the assumption.

$$
\left[\Sigma \Sigma^{n} W, \Sigma^{n} Y\right] \stackrel{i_{\Sigma^{n} f_{*}}^{\longrightarrow}}{\longrightarrow}\left[\Sigma \Sigma^{n} W, \Sigma^{n} Y \cup_{\Sigma^{n} f} C \Sigma^{n} X\right] \stackrel{q_{\Sigma^{n} f_{*}}}{\longrightarrow}\left[\Sigma \Sigma^{n} W, \Sigma^{n+1} X\right]
$$

For a space $U$ and its subspace $V$ with $V \ni *$, we set $\Omega(U, V)=\operatorname{Map}(I, 0,1 ; U, V, *)$, the space of maps $\omega: I \rightarrow U$ such that $\omega(0) \in V$ and $\omega(1)=*$ with compact open topology. Note that $\Omega(U, *)=\Omega U$, the loop space of $U$. For any space $Z$, we have the exact sequence

$$
\left[Z, \Omega \Sigma^{n} Y\right] \stackrel{\Omega i_{\Sigma^{n}} f_{*}}{\longrightarrow}\left[Z, \Omega\left(\Sigma^{n} Y \cup_{\Sigma^{n} f} C \Sigma^{n} X\right)\right] \stackrel{j_{*}}{\longrightarrow}\left[Z, \Omega\left(\Sigma^{n} Y \cup_{\Sigma^{n} f} C \Sigma^{n} X, \Sigma^{n} Y\right)\right],
$$

where $j: \Omega\left(\Sigma^{n} Y \cup_{\Sigma^{n} f} C \Sigma^{n} X\right) \rightarrow \Omega\left(\Sigma^{n} Y \cup_{\Sigma^{n} f} C \Sigma^{n} X, \Sigma^{n} Y\right)$ is the inclusion map

$$
\operatorname{Map}\left(I, 0,1 ; \Sigma^{n} Y \cup_{\Sigma^{n} f} C \Sigma^{n} X, *, *\right) \rightarrow \operatorname{Map}\left(I, 0,1 ; \Sigma^{n} Y \cup_{\Sigma^{n} f} C \Sigma^{n} X, \Sigma^{n} Y, *\right)
$$

(cf. [AGP, 3.3.24]). Set $\ell=\operatorname{conn}\left(Y \cup_{f} C X, Y\right)+\operatorname{conn}(Y)$. Then $\operatorname{conn}\left(\Sigma^{n} Y \cup_{\Sigma^{n} f}\right.$ $\left.C \Sigma^{n} X, \Sigma^{n} Y\right)+\operatorname{conn}\left(\Sigma^{n} Y\right) \geq 2 n+\ell$ by the assumption (1.2) and the Whitehead theorem [tD, (20.6.3)]. Consider the commutative square

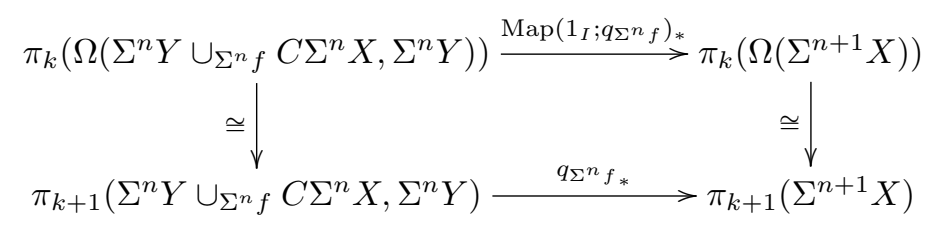

where $\operatorname{Map}\left(1_{I} ; q_{\Sigma^{n} f}\right): \operatorname{Map}\left(I, 0,1 ; \Sigma^{n} Y \cup_{\Sigma^{n} f} C \Sigma^{n} X, \Sigma^{n} Y, *\right) \rightarrow \operatorname{Map}\left(I, 0,1 ; \Sigma^{n+1} X, *, *\right)$ is the map induced from $q_{\Sigma^{n} f}$. Since $q_{\Sigma^{n} f_{*}}$ of the square is bijective for $k<2 n+\ell$ and surjective for $k=2 n+\ell$ by a theorem of Blakers-Massey [tD, (6.10.2)], it follows that $\operatorname{Map}\left(1_{I} ; q_{\Sigma^{n} f}\right)$ is $2 n+\ell$-connected so that

$$
\operatorname{Map}\left(1_{I} ; q_{\Sigma^{n} f}\right)_{*}:\left[Z, \Omega\left(\Sigma^{n} Y \cup_{\Sigma^{n} f} C \Sigma^{n} X, \Sigma^{n} Y\right)\right] \rightarrow\left[Z, \Omega \Sigma^{n+1} X\right]
$$

is bijective if $Z$ is a $\mathrm{CW}$-complex with $\operatorname{dim} Z<2 n+\ell$ (cf. [tD, (8.4.2)]). Hence $\operatorname{Map}\left(1_{I} ; q_{\Sigma^{n} f}\right)_{*}:\left[\Sigma^{n} W, \Omega\left(\Sigma^{n} Y \cup_{\Sigma^{n} f} C \Sigma^{n} X, \Sigma^{n} Y\right)\right] \rightarrow\left[\Sigma^{n} W, \Omega \Sigma^{n+1} X\right]$ is bijective by the assumption (1.2). The composite of

$$
\begin{gathered}
{\left[\Sigma^{n} W, \Omega\left(\Sigma^{n} Y \cup_{\Sigma^{n} f} C \Sigma^{n} X\right)\right] \stackrel{j_{*}}{\longrightarrow}\left[\Sigma^{n} W, \Omega\left(\Sigma^{n} Y \cup_{\Sigma^{n} f} C \Sigma^{n} X, \Sigma^{n} Y\right)\right]} \\
\frac{\operatorname{Map}\left(1_{I} ; q_{\Sigma^{n} f}\right)_{*}}{\cong}\left[\Sigma^{n} W, \Omega \Sigma^{n+1} X\right]
\end{gathered}
$$

is $\Omega q_{\Sigma^{n} f_{*}}$. Hence the sequence

$$
\left[\Sigma^{n} W, \Omega \Sigma^{n} Y\right] \stackrel{\Omega i_{\Sigma^{n}}{ }^{*}}{\longrightarrow}\left[\Sigma^{n} W, \Omega\left(\Sigma^{n} Y \cup_{\Sigma^{n} f} C \Sigma^{n} X\right)\right] \stackrel{\Omega q_{\Sigma^{n} f_{*}}}{\longrightarrow}\left[\Sigma^{n} W, \Omega \Sigma^{n+1} X\right]
$$


is exact so that the sequence (5.1) is exact. It follows from Corollary 3.8(2) that any $\widehat{\gamma} \in\left[\Sigma^{n+1} W, \Sigma^{n} Y \cup_{\Sigma^{n} f} C \Sigma^{n} X\right]$ with $q_{\Sigma^{n} f_{*}} \widehat{\gamma}=-\Sigma^{n+1} \gamma$ is in $\operatorname{Coext}_{\Sigma^{n} f}\left(\Sigma^{n} \gamma\right)$. If additionally $2 \cdot \operatorname{conn}(Y) \geq \operatorname{dim} W$, then $\Sigma^{n}:[\Sigma W, Y] \rightarrow\left[\Sigma^{n+1} W, \Sigma^{n} Y\right]$ is surjective so that $\widehat{\gamma} \in \operatorname{Coext}_{f}^{n}(\gamma)$ by Proposition 3.7(2). This completes the proof of Proposition 1.2 .

\section{6. $\quad$ Proof of Corollary 1.3}

The following problem is a part of Problem I.

Problem 6.1. Given a map $f: X \rightarrow Y$, is the canonical homeomorphism

$$
\varphi: \Sigma\left(Y \cup_{f} C X\right) \rightarrow \Sigma Y \cup_{\Sigma f} C \Sigma X, y \wedge \bar{t} \mapsto y \wedge \bar{t}, x \wedge s \wedge \bar{t} \mapsto x \wedge \bar{t} \wedge s
$$

homotopic to a coextension of $q_{f}: Y \cup_{f} C X \rightarrow \Sigma X$ w.r.t. $\Sigma f: \Sigma X \rightarrow \Sigma Y$ ?

Corollary 1.3 is a partial answer to Problem 6.1 .

Proof of Corollary 1.3. Let $k \geq 3$ or $k=m=2$. Let $n=0, W=M_{m}^{k}=$ $\mathrm{S}^{k-1} \cup_{m \iota_{k-1}} C \mathrm{~S}^{k-1}, X=Y=\mathrm{S}^{k}, f=m \iota_{k}, g=q_{m \iota_{k-1}}$. If $k \geq 3$, then $W \approx \Sigma M_{m}^{k-1}$ so that the group $\left[\Sigma W, Y \cup_{f} C X\right]$ is commutative. If $k=m=2$, then we can prove that $\left[\Sigma W, Y \cup_{f} C X\right] \cong \mathbb{Z}_{4}$ by virtue of $[\mathbf{T 3}$, Theorem 4.1]. Therefore (1.2) of Proposition 1.2 is satisfied and so $\varphi_{m}^{k}$ is homotopic to a coextension of $q_{m \iota_{k-1}}$ w.r.t. $m \iota_{k}$.

\section{References}

[AGP] M. Aguilar, S. Gitler and C. Prieto, Algebraic topology from a homotopical viewpoint, UTX, Springer, 2002.

[tD] T. tom Dieck, Algebraic topology, EMS Textbooks in Math., European Math. Soc., 2008.

[J] I. M. James, On the homotopy groups of certain pairs and triads, Quart. J. Math. 5 (1954), 260-270.

[Og] K. Ôguchi, A generalization of secondary composition and its applications, J. Fac. Sci. Univ. Tokyo 10 (1963), 29-79.

[P] D. Puppe, Homotopiemengen und ihre induzierten Abbildungen I, Math. Z. 69 (1958), 299-344.

[T1] H. Toda, Generalized Whitehead products and homotopy groups of spheres, J. Inst. Poly. Osaka City Univ.3 (1952), 43-82.

[T2] H. Toda, Composition methods in homotopy groups of spheres, Ann. of Math. Studies 49, Princeton, 1962.

[T3] H. Toda, Order of the identity class of a suspension space, Ann. of Math. 78 (1963), 300-325. 